\title{
Estudos dos micropezídeos da Bacia Amazônica

\author{
I - Contribuição à Sistemática de Micropezidae e estudo do gênero Taeniaptera \\ Macquart, 1835 (diptera - acalyptratae) (*)
}

Lindalva Paes de Albuquerque ${ }^{* *}$ )

\begin{abstract}
Resumo
Este trabalho é uma avaliaçăo da sistemática dos Micropezídeos com vista à posição da familia Micropezidae, estendendo-se à subfamilia Taeniapterinae. Inicia-se o estudo do gênero Taeniaptera pelas espécies que ocorrem na Amazônia Centrat, com base nos caracteres dif̃erenciais da genitália dos machos e das fêmeas A identificação dos exemplares fez-se com auxilio da chave de Henning (1934) e comparando os caracteres desses exemplares com a descrição original de cada espécie e com material identificado pelo dipterologista Dr. H. S. Lopes. Fez-se também estudo da morfologia, anatomia da genitália e distribuição da espécie Teniaptera iasciva (Fabr. 1798). Essa espécie apresenta célula posterior da asa fechada e pedúnculada e o quinto esternito do macho simples.
\end{abstract}

\section{INTRODUÇÃo}

Nosso interesse pelo estudo dos Micropezideos deve-se ao fato de ser uma família em sua maioria neotropical. Pelas espécies reteridas na bibliografia que ocorrem na Amazônia, pelo material coletado durante nossos trabalhos, pelas condiçc̃es ecológicas, acredita-se que a região venha a ser conhecida como uma área onde se encontra a maior diversidade desses dipteros.

Através deste trabalho, procura-se ampliar o conhecimento dos Taeniaptera, com interes. se na taxionomia de espécies da Amazônia Central.

$\mathrm{Na}$ chave de identificação de Hennig, 1934:74-77, a qual se considera a mais completa para o gênero estão contidas 36 espécies de Taeniaptera.

De acordo com Steyskal (1968) o gênero conta com 41 espécies, 22 das quais assinala- das no Brasil, sendo que 19 destas ocorrem na região Amazônica.

$\mathrm{Na}$ descrição das espécies de Taeniaptera foram utilizados caracteres da morfologia externa, quetotaxia, cores estruturais e comparações dimensionais dos segmentos. Mas a base fundamental desse trabalho, sob o aspecto sistemático, prende-se ao estudio comparativo da anatomia da genitália dos machos e das fêmeas, onde há caracteres que permitem determinações ao nivel de espécie e concluir com segurança as afinidades existentes entre os grupos.

O gênero Taeniaptera retém o maior núme ro de caracteres primitivos dos Micropezídeos (Aczel, 1951). Pretende-se através da seçueência de estudo dos diversos gêneros, adquirir maiores conhecimentos da biologia e processos evolutivos do grupo.

\section{MATERIAL E MÉTODOS}

Esses dípteros toram coletados nos mais variados locais como: Mata alta, Capoeira, Campina, Campo e Várzea. Utilizando-se rede entomológica e aspirador, quando coletados diretamente na vegetação; e pequenas reảes quando atraidos por isca de fezes humana, carne bovina e de peixe em decomposição e frutos silvestres. Para coletas noturnas empregaramse iscas luminosas de luz fluorescente com lâmpada GE luz negra e luz do dia, e lâmpacia de mercúrio. Incluiram-se às observações, exemplares da Coleção do Museu Paraense Emílio Goeldi, Belém, o material básico às icien-

( *) - Parte da tese submetida ao Curso de Pós-Graduação do Instituto Nacional de Pesquisas da Amazônia (INPA) e Fundação Universidade do Amazonas (FUA), para obtenção do grau de Magister Scientae, em 1979.

("*) - Instituto Nacional de Pesquisas da Amazônia e Universidade do Amazonas, Manaus. 
tificações compreende exemplares identificados pelo dipterologista H. S. Lopes entre 1964 a 1968 .

$\mathrm{Na}$ impossibilidade de obter-se os "Tipos", fez-se as redescrições das espécies conhecidas comparando-as com a descrição original, observações microscópicas da morfoiogia de exemplares disponíveis e estudo anatômico da genitália dos machos e fêmeas, onde há caracteres muito seguros ao nível específico.

A terminologia empregada na descrição das espécies quanto à morfologia externa é a de Cresson (1930), e para a anatomia da genitália é a de Hennig (1934-1936), com ligeiras modificações.

A dissecação, montagem de material a seco e preparação de lâminas segue o método clássico em Entomologia para estudos morfológi$\cos$. As lâminas resultantes desse estudo, juntamente com o material a seco e os exemplares no álcool a $70 \%$, estão depositados na Coleção Entomológica do INPA, como documentaçãc deste trabalho.

\section{Resultados}

POSIÇÃO DA FAMÍLIA MICROPEZIDAE

A sistemática os Diptera-Micropezidae, apresentada por (Enderlein, 1922; Frey, 1927 e Cresson, 1930 e 1938) fornece consideráveis informações, especialmente sobre o parentesco das famílias Paleárticas, Neárticas e Neotropicais.

O conhecimento dos Micropezídeos e suas interrelaçöes filogenéticas, tornam-se mais substanciais com os trabalhos de Hennig (19341938), cujos estudos baseiam-se também na morfologia da genitália.

Aczel (1949-1951) faz uma revisão das espécies de Tylidae (Micropezidae). Aczel, em 1949 b, publicou um Catálogo das espécies de Tylidae encontradas na região Neotropical: fez a revisão dos Tilídeos usando as bases taxionômicas de Hennig (1938), propõe o nome de TANYPEZIDIFORMES para o grupo natural que abrange as famílias de Tanypezidae, Tyliciae e Neriidae (Fig. 1).

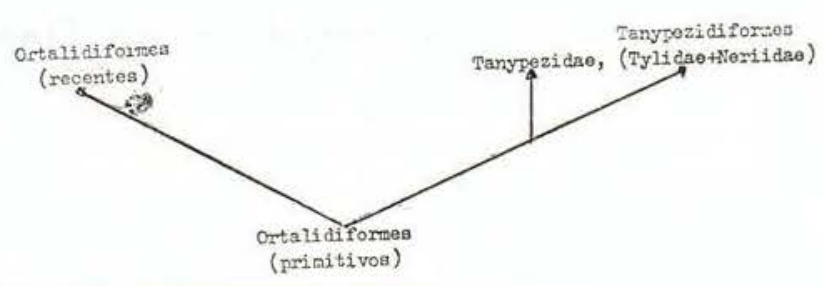

Fig. 1 - Esquema da evolução dos grupos (Aczel 1949)

Para Aczel (1949a) a família Tylidae (Micropezidae) pode ser dividida em subfamílias, segundo os autores (Quadro 1 esquema adap. tado) .

Cresson (1930) faz uma breve revisão dos Micropezideos estudando as espécies Neotro picais. Desde então a família foi reconhecida para as espécies americanas que foram ligeiramente tratadas por Hennig $(1935,1936) \mathrm{em}$ sua Revision der Tyliden, valiosa e indispensável contribuição ao estudo desse grupo.

Hendel (1922) foi o primeiro a elevar Neriinae a família independente de Tylidae (-Micropezidae) .

Enderlein (1922), em sua Klassifikation der Micropeziden, inclui Neriidae como subfamília de Tylidae, assim como os outros autores. Tendo considerável material da América do Sul. deu pouca ênfase às espécies paleárticas e neárticas e manteve Neriinae e Micropezinae.

QUADRO I - Divisão da sistemática segundo diferentes autores

\begin{tabular}{l|l|l}
\hline Hennig (1938) & Gresson (1930-38) & Outros autores \\
\hline $1-$ Tylidae & $1-$ Micropezidae & - \\
\hline $\begin{array}{l}\text { 1. Taeniapterinae } \\
\text { (Tanypodinae, } \\
\text { Rainerinae, } \\
\text { Czerny, 1930) }\end{array}$ & 1. Taeniapterinae & \\
$\begin{array}{l}\text { 2. Trepidarinae } \\
\text { (Calobatinae) }\end{array}$ & 2. Calobatinae & 1 + 2 Calobatidae \\
$\begin{array}{l}\text { 3. Tylinae } \\
\text { (Micropezinae) }\end{array}$ & 3. Micropezinae & 3. Micropezidae \\
$\begin{array}{l}\text { 4. Neriinae } \\
\text { 4. Neriidae }\end{array}$ & 4. Neriidae \\
\hline
\end{tabular}


Frey (1927) discute a conveniência de manter Neriidae independente de Tylidae. Para o autor, a semelhança entre os dois grupos é somente um fenômeno de convergência.

Para Aczel (1951) as famílias de Tanypezidiformes são facilmente diferenciáveis, acha que as formas intermediárias entre os grupos se extinguiram há longo tempo, o que reforça o conceito de relíquia dos Tanypezidiformes entre os grupos de Acalyptrata.

Hennig (1973) considera Taeniapteridae. além de (Colobalidae [Trepidariidae]) e Micropezidae (Tylidae), uma família de Micropezoidea. Inclui nesta familia também Lypselosomatidae, Pseudopomyzidae e Neriidae.

Rohdendorf (1974) inclui os Micropezidae (Neriinae, Trepidariinae e Taeniapterinae) na super família Psiloidae.

Os caracteres mais comumente usados até agora necessitam de estudo quanto ao seu valor taxionômico, pois de sua variabilidade específica pouco se conhece.

\section{CARACTERIZAÇÃo DA FAMÍLIA}

A familia Micropezidae Loew, 1862 foi colocada na Lista Oficial de Grupos dos Nomes de Famílias em Zoologia. Direction 41 Inst. Com. Zool. Nom., em 1956.

Para Cresson (1930 e 1938), Hennig (1934, 1938) e Aczel (1951), Micropezidae caracteriza-se por apresentar: - tamanho moderado, corpo geralmente esguio, pernas longas; terceiro segmento antenal achatado lateralmente, decaído, arista dorsal com implantação subbasal; - pro-tórax retraído, meso-tórax desenvolvido o que determina grande distância entre as patas anteriores e médias; - patas anteriores mais curtas que as demais; - costa da asa sem quebradura; - ápice da célula anal reto; - machos usualmente com lobos progenitais no quinto esternito; - armadura genital bem diferenciada dos grupos próximos; clasperes quando presentes, bem desenvolvidos, separados e distintamente deslocados dos segmentos genitais - sexto esternito ventral e sétimo lateral;
Os caracteres aqui destacados, possibilitam separar as subfamílias: Taeniapterina, Calobatinae e Micropezinae.

\section{SUBFAMÍLIA TAENIAPETERINAE}

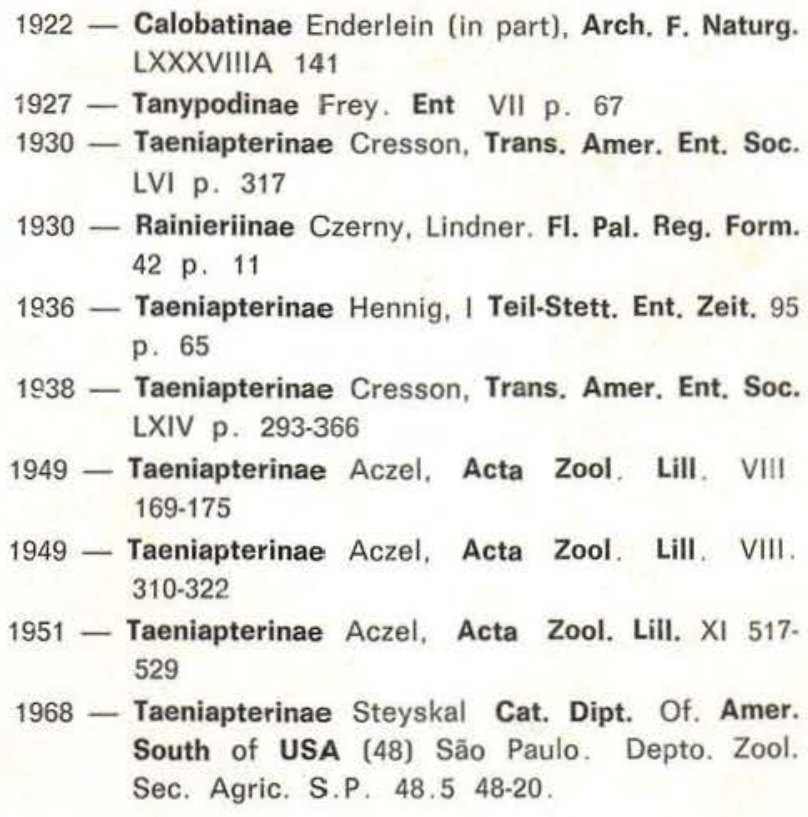

Cresson (1938) considera que Enderlein (1922) não teve conceituação lógica das duas subfamílias; e acrescenta que Taeniapterinae tem prioridade sobre Rainierinae; e que Calobaninae e Micropezinae têm limites muito estreitos, ocasionando dificuldades na separação dos dois grupos, enquanto Taeniapterinae não é difícil separar das duas, sobretudo pelas características da genitália do macho, com ajuaja de caracteres morfológicos como:

Cabeça - subgiobosa; estria frontal fusiforme; lâmina ocelar no meio da fronte, parafaciália pouco evidente; frontália atenuada, anteriormente alongada na porção mediana; face mais larga que longa; antenas inseridas longe uma da outra;

Tórax - estreito e aiongado anteriormente; capitulo do balancim compacto; um par de cerdas bem desenvolvidas na margem anterior da esternopleura, cerdas hipopleurais presentes;

Abdômen - segmentos lateralmente comprimidos cláspers situados abaixo do quinto esternito; processo copulatório formado com 


$\begin{array}{llll}\text { Caracteres } & \text { Taeniapterinae } & \text { Calobatinae } & \text { Micropezinae }\end{array}$

\section{I-Cabeça}

1. Forma

2. Fronte

3. Abertura bucal

4. Anteclípeo

5. Palp. maxila

6. Premento

I-Tórax

7. Porção anterior

8. Escutelo

9. Esternopleura

10. Cerdas torácicas:
a) propleurais
b) dorsais
c) notopleurais
d) esternopleurais
e) hipopleurais

11. Asas

a) nervuras e células:

$$
\begin{aligned}
& a_{1}-\mathrm{M}_{3} \\
& a_{2}-r_{4}+\overline{5}^{-m} \\
& a-\text { estigma alar } \\
& 3 \\
& a-\text { célula anal } \\
& 4
\end{aligned}
$$

b) manchas

12. Balancim

13. Pernas
a) anéis
b) tarsos: anterior

$\begin{array}{ll}\text { - globosa } & \text { - alongada } \\ \text { - larga e convexa ou plana } & \text { - estreita } \\ \text { - pequena, ovalada } & \text { - arredondada } \\ \text { - largo, amarelo } & \text { - pequeno } \\ \text { - claviformes } & \text { - subcilindricos } \\ \text { - largo } & \text { - quadrangular }\end{array}$

- alongada

- normal

- normal

- em V prolongado

- normal

- ausente

$-0-3$ pares

$-1-2$ pares

-1 par

$-2-3$ grandes

$-2-3$ curtas

— alongadas, alula grande, —alongada, alula pequena, incisão reduzida

- alongadas, alula peque na, incisão reduzida

$$
\begin{aligned}
& \text { - ausente } \\
& \text { - convergentes ou fusio- } \\
& \text { nadas } \\
& \text { - ausentes } \\
& \text { - curta } \\
& \text { - ausente }
\end{aligned}
$$$$
\text { - ausentes }
$$$$
\text { - capítulo lobuloso gran }
$$
de, pedúnculo pequeno.$$
\text { - menores e finas }
$$$$
\text { - escuros não estruturais }
$$$$
\text { - variáveis }
$$

\section{III - Abdômen}

14. Pré-abdômen longo estreito:
a) $\mathrm{VI} T$
- maior que o $\mathrm{V} \mathrm{T}$
- igual ao $\mathrm{V} \mathrm{T}$
- menor que o $\mathrm{V} \mathrm{T}$
b) epandrium
- longo, comprido, estável
- curto
- curto
c) abertura ventral
- linear estreita
- larga
- larga
d) sulco VII + VIII T - vestigial
- rudimentar
- rudimentar 
base cilíndrica; porção distal do quinto esternito bífida, digitiforme e em grande parte com processos secundários dorsais.

De acordo com Hennig (1934) e Cresson (1938), a subfamília Taeniapterinae tem caracteres que se pode considerar como os mais relevantes: estria frontal fusiforme; lâmina ocelar na posição mediana; antenas afastadas entre si; capítulo do balancim compacto e processo copulatório usualmente presente com a base formada por um tronco comum.

\section{Gênero Taeniaptera}

1835 - Taeniaptera Macquart Hist. Nat. Dipt. 2 (Suites a Buffon Dipt. 2): 41 espécie tipo triviatatta Macquart

1850 - Grallomya Rondani, 1 c. (3) 2: 180 Espécie tipo Calobata tarsata Wiedemann (des. orig.)

1850 - Grallopoda Rondani, Nuov. Ann. Sci. Nat. Bologna (3) 2: 178 Espécie tipo Calobata albimana Macquart (des. orig.) = trivittata Macquart.

1922 - Grallopoda Enderlein, Arch. F. Naturg. 88A. 5: 212

1927 - Grallomoya Frey, Notulae Ent. 7: 6, 8, 72

1930 - Taeniaptera Cresson, Trans. Amer. Ent. Soc. 56: 349

1930 - Grallomyia Cresson, 1 c. 56: 337-338

1933a- Grallomyia (Neograllomyia) Hendel, Rev. de Ent. R. de Janeiro 3: 65 Espécie tipo longivitta Hendel (des. orig.) = vittipenis (Coquillett)

1933a- Grallomyia (Paragrallomyia) Hendel Rev. de Ent. R. de Janeiro 3: 63 Espécie tipo Musca annulata Fabricius (des. orig.)

1934 - Taeniaptera Hennig Stetting. Ent. Zeigt, 95: 73-77

1938 - Teniaptera Cresson, Trans. Amer. Ent. Soc. LXIV 347

1949 - Taeniaptera Aczel, Acta Zool. Lill. VIII: 312 e 359

1951 - Taeniaptera Aczel, Acta Zool. Lill. XI: 517-529

1968 - Taeniaptera Steyskal, Cat. Dipt. of Amer. South of USA (48) S. Paulo. Dep. Zool. Sec. Agric. S.P. $48, .5-48.20$

O gênero Taeniaptera foi criado por Macquart (1835:491) para abrigar a espécie $T$. trivitatía Macquart.

Cresson (1930) considera como Taeniaptera aqueles indivíduos com a fronte mais ou menos enrugada e com pequena mancha escura, preta e aveludada na porção mediana da fronte; e Grallomya os que apresentam testa lisa e parafrontália larga.
Hennig (1934) diz que o maior ou menor afastamento das veias $r$ e $m$ foi usado por Macquart, Rondani, Enderlein e Frey como o único caráter que distingue Taeniaptera de Grallomyia.

Cresson (1938) considera Grallopoda como sinônimia estricta de Taeniaptera, porque tem como espécie tipo, T. tarsata (Wiedemann).

As espécies de Grallomyia Enderlein p.part. (nec) Rondani, passaram então a ser chamadas por Hennig de Poecilomyia, um novo gênero que depois foi substituído por Poecilotylus, pelo fato de Poecilomyia ser um nome preocupado por (Hendel, 1911). Poecylotylus caracteriza-se pelos palpos regularmente cilíndricos, mesofronte alargada e longa estendendo-se para trás do nível da testa. Ao contrário Taeniaptera caracteriza-se por apresentar, segundo Hennig (1936) e Cresson (1938), essencialmente: cabeça livre, subgiobosa, mais alta que larga, com olhos verticais; mesofronte plana, fusiforme, com pequenas manchas escuras e anterior a lâmina ocelar; fronte algumas vezes enrugada e vértex carenado; palpos regularmente espalmados com margem anterior clara; setas da cabeça que podem faltar: pósverticais (pvt), verticais externas (vte) e orbitais superiores (orss); terceiro segmento da antena alargado, arista nua; tórax castanho vermelho ou azulado que pode ser brilhante ou opaco; calo umeral algumas vezes carenado; cerdas dorsocentrais de 0-4 presentes; escutelo curto pubescente, não plumoso; patas escuras com um ou dois anéis bráncos no fêmur; asas com três faixas transversais cinza, uma basal estreita, uma larga mediana e uma apical arredondada, podem ser mais largas ou desaparecer em parte; primeira faixa sub-basal desaparecendo. Ou um cinza esfumaçado uniforme, ainda pode ter uma faixa longitudinal mediana.

De posse desses caracteres é mais fácil distinguir as espécies de Taeniaptera dos demais grupos.

\section{Teaniaptera lasciva (Fabricius, 1798)}

Musca lasciva Fabricius, 1798:564

Calobata lasciva Fabricius, 1805:262

Calobata ruficeps Guérin, 1829:1103 
Calobata aloa Walker, 1849:1053

Grallopoda annulata Rondani, 1850:178

Calobata lasciva Johnson, 1894:336

Calobata annulata Wulp, 1897:372

Calobata lasciva Coquillett, 1900:257

Calobata lasciva Cresson, 1908:10

Grallopoda lasciva Enderlein, 1922:212

Grallopoda lasciva var, albimana Enderlein, 1922:212

Grallopoda lasciva Frey, 1927:72

Taeniaptera lasciva var, obliterata Cresson, 1930:351

Grallopoda lasciva Czerny, 1932:281

Taeniaptera lasciva Fischer, 1932:21

Taeniaptera lasciva Hennig, 1934:86-88

Taeniaptera lasciva Aczel, 1949:316

Taeniaptera lasciva Aczel, 1951:517, 523-527

Taeniaptera lasciva Steyskal, 1968:48:18

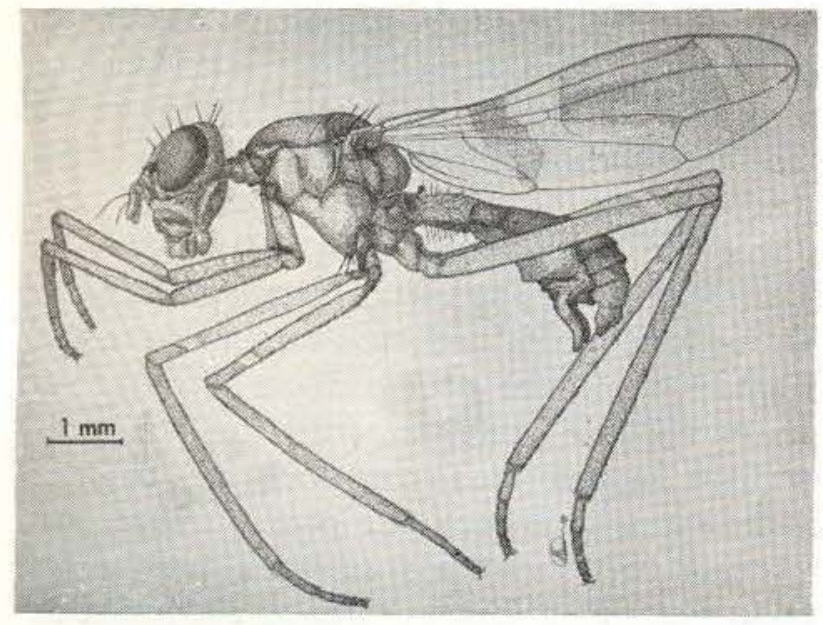

Fig. 2 - Foto de Taeniaptera lasciva (Fabricius, 1798).

мACHO - Comprimento em média $9,8 \mathrm{~mm}$. Coloração básica castanho-escuro.

Cabeça - (Fig. 3) subglobosa, mais larga que longa. Mesofronte bem delimitada castanho-escura brilhante, tornando-se mais clara na porção anterior. Lâmina ocelar castanho-escura, ocelos claro. Anterior à lâmina ocelar, mancha castanha enegrecida que se estreita e se torna mais clara próximo à borda anterior da mesofronte. Frontália castanho-brilhante. Parafrontália rugosa, castanho-clara e pruinosidade prateada; Epicéfala castanho ligeiramente protuberante e rugosa, com reflexos prateados. Paracéfala castanho com pequenas setas e pêlos curtos se estendem pelo occiput castanhoescuro. Um par de setas bem desenvolvidos próximo à inserção do pescoço. Gena castanho, densa, pruinosidade prateada e seis pequenas setas próximo à abertura bucal e pêlos adicionais. Lâmina mesofacial castanho-ciara, borda da abertura bucal castanho-escura, com micropêlos. Antena: esclerito basal castanho-briIhante; primeiro e segundo segmentos castanho-claros com pequenas setas superficiais e marginais; terceiro segmento, o mais desenvolvido, coberto de pilosidade clara; arista, de base clara escurecendo distalmente. Fossa antenal castanho brithante e pruinosidade clara. Peças bucais: anteclípeo castanho-brilhante. Palpos maxilares escuros, com margem anterior clara. Labelo claro com finos pelos superficiais. Premento de forma quadranguiar, castanho-escuro brilhante com pêlos superficiais que se condensam na margem anterior.

Setas da cabeça: 2 pares orsa, o primeiro ligeiramente reclinado $€$ o segundo procliriado; 1 par orss, ligeiramente reclinado; 1 par vte, divergente; 1 par vti, fracamente proclinado e 1 par pvt, paraielo.

Tórax - Superfície dorsal cinza, coberta de pruinosidade de reflexos dourados, que se estende até o escutelo. Calo umerai com mancha castanho-clara e pequenas setas na mar-

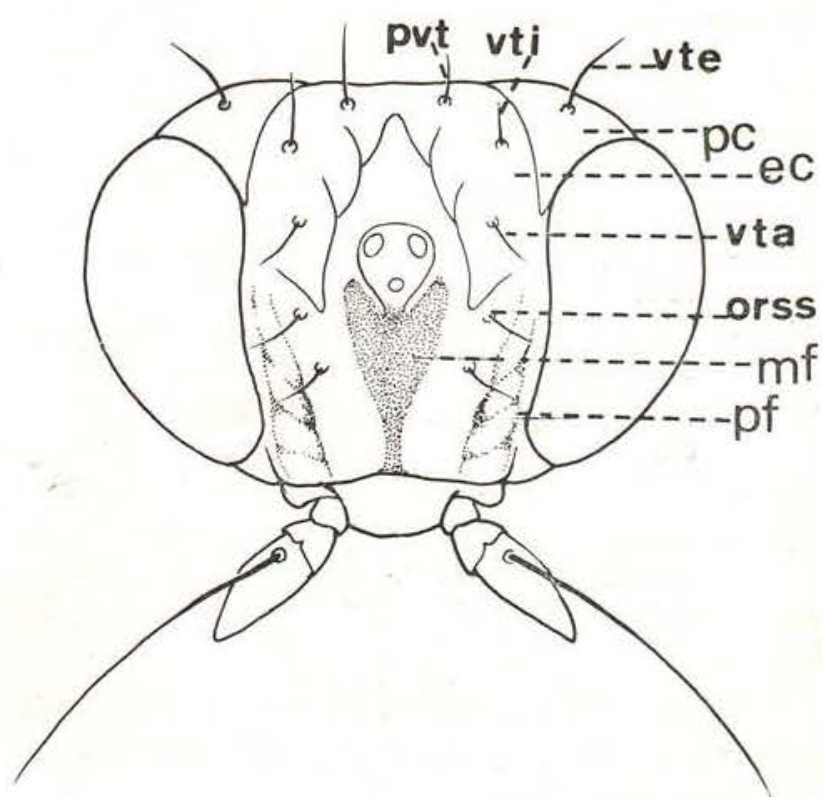

Fig. 3 - Cabeça de T. lasciva. lidentificação segundo Hennig 1934. Áreas: $p c=$ paracefala; $e c=$ epicefala; $\mathrm{mf}=$ mesofronte; $\mathrm{pf}=$ parafrontália. Cerdas: pvt $=$ pós verticais; $v t i=$ verticais internas; orss = orbitais superiores; vta $=$ verticais anteriores; vte $=$ verticais externas. 


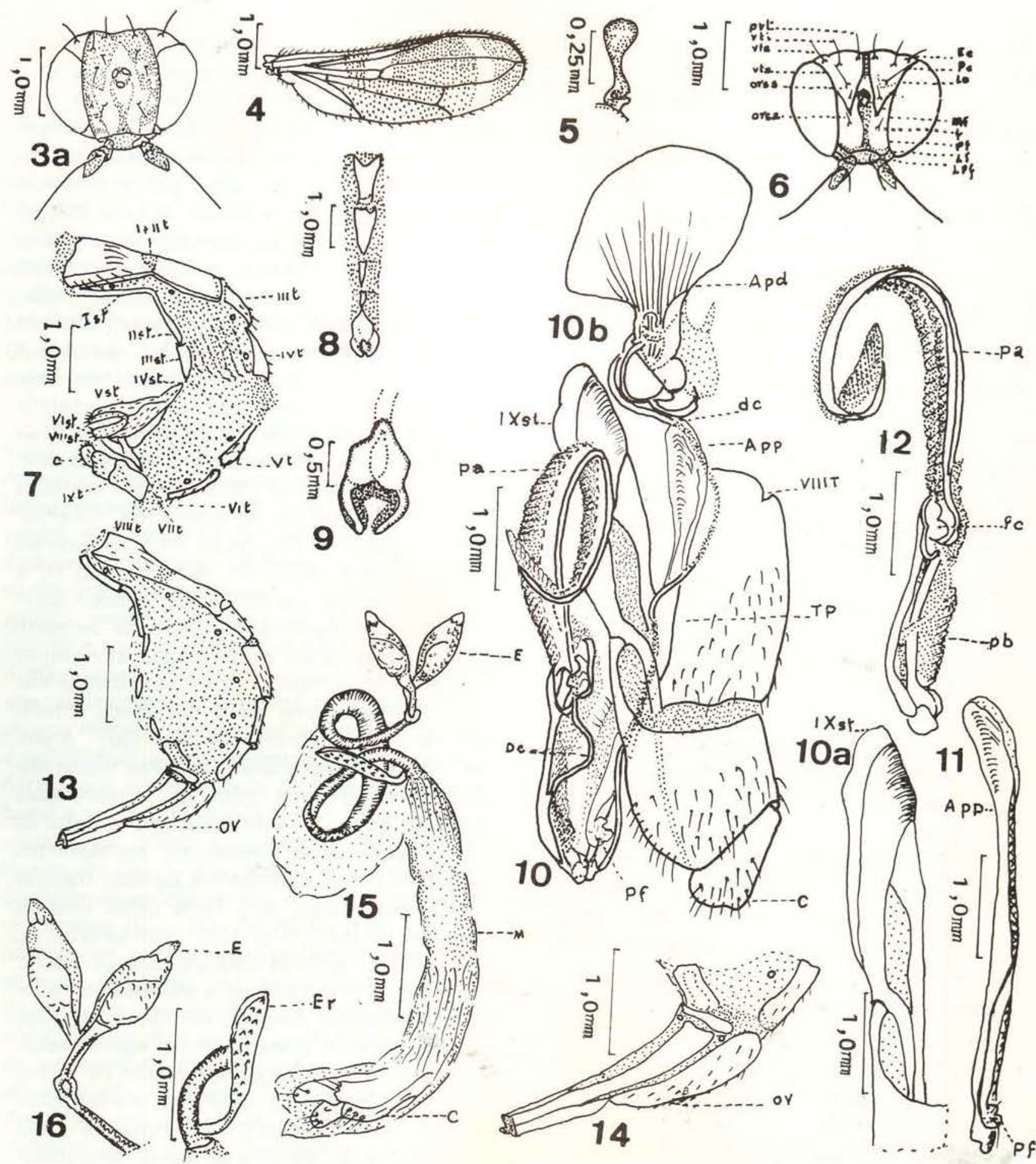

Fig. 3a/16 - Taeniaptera lasciva - Macho: 3a) - cabeça; 4) - asa; 5) - halter; 6) - cabeça (identificação das áreas: $\mathrm{Ec}=$ epicefalo; $\mathrm{Pc}=$ paracefalo; $\mathrm{Lo}=$ lâmina ocelar; $\mathrm{Mf}=$ mesofronte; $\mathrm{F}=$ fronte; $\mathrm{Pf}=$ parafrontália; Lf = lâmina facial; Lpf = lâmina parafacial); 7) - abdômen; 8) - esternitos de I-VII; 9) — esternito V; 10) — genitália; 10a) - placa ventral do IX esternito; 10b) - Apodema do duto; 11) — apodema do edeago; 12) - edeago Fêmea: 13) - abdômen; 14) - avipositor; 15) - genitália; 16) - espermatecas (Apd = apodema do duto; App = apodema do edeago; $\mathrm{De}=$ duto ejaculatório; $\mathrm{TP}=$ teca do edeago; $\mathrm{Pf}=$ pinça fálica; $\mathrm{Pb}=$ porção basal; $\mathrm{Fc}=$ formação capsular; $\mathrm{Pa}=$ porção apiceal; $\mathrm{E}=$ espermatecas; $\mathrm{Er}=$ espermateca rudimentar; $\mathrm{M}=$ membrana; $\mathrm{C}=$ cerci; $\mathrm{Dc}=$ duto comum; I $\mathrm{t}-\mathrm{IX} \mathrm{t}=$ primeiro ao nono tergito; $\mathrm{I}-\mathrm{VII}$ st. = primeiro ao sétimo esternito; IX st. $=$ nono esternito).

Estudos... 
gem inferior. Pleuras castanho-enegrecidas com reflexos intercalados de pruinosidade castanha e prateada. Pós-escutelo ligeiramente protuberante, com pruinosidade prateada que se condensa na porção inferior formando duas áreas de reflexos mais intensos. Setas do tórax: 6 a 8 setas pro-pleurais; 2 pares notopleurais subiguais; 1 par supra-alar; 1 para pósalar paralelo; 1 par dorso-central ligeiramente reclinado; 1 par escutelar apical fracamente convergente; pente esternopleural formado de 13 setas escuras, bem desenvolvidas e setas menores claras e escuras adicionais; próximo à inserção do segundo par de patas, 6 setas enfileiradas e pêlos menores, claros. Pernas: coxa I, II e III, castanhas, cobertas de pruinosidade prateada e pequenas setas marginais. Perna I: fêmur e tíbia igualmente castanhos, tarso basal e apical escuros e os três restantes brancos. Pernas II: fêmur castanho com anel subapical branco de largura $1 / 1$ da largura do fêmur castanho-claro, tíbias e tarso igualmente castanhos. Pernas Iil: fêmur com anel basal e subapical branco, ápice castanho-claro, tíbia e tarsos igualmente castanhos. Asa (Fig. 4) em média com $7,40 \mathrm{~mm}$ de comprimento por $2,40 \mathrm{~mm}$ de largura, base hialina e mancha escura transversal na fusão da sobcostal. Área hialina duas vezes a largura da mancha escura anterior, metade apical da asa escura e interrompida apenas por uma faixa estreita hiaiina subapical em meia lua, que se inicia na extremidade da $\mathbf{r}_{1}$ e toca a margem oposta da asa Halter conforme a Fig. 5. Cabeça com areas identificadas na Fig. 6.

Abdômen - (Fig. 7) cor básica castanhoescura. Os dois primeiros tergitos formando uma peça única. com pêlos longos, finos $€$ claros, distribuídos na superfície e condensandose nas margens laterais e posterior; onde há pêlos escuros. A fusão dos dois primeiros tergitos evidencia-se por um sulco vestigial $e$ intensa pruinosiciade que se estende ao terço distal do segmento I atingindo larga faixa do segmento II. Borda posterior do tergito III e IV, membranosa, clara e marcada por uma fileira de pequenas setas marginais. $\mathrm{O}$ segmento VI é o menor de todos. Esternitos (Fig. 8); esternito I alargado na base, estreitanao-se progressivamente até a junção com o esternito
II de forma triangular; esternitos III e IV reduzidos a estreita trave; esternito $V$ desenvolvido com pequenas setas superficiais e extremidade distal bifurcada, ápice das bifurcações convergentes, face interna forrada de robustos espinhos e fina setas, que se condensam na extremidade distal. Apófises basais ausentes (Fig. 9) . Esternito VI estreito na base que se insere junto à base do esternito $V$ servindothe de suporte, alargando-se para a margem distal onde se une ao esternito VII, rudimentar. estreitc, de posição transversal; a extremidade esquerda vai unir-se ao tergito VII, reduzido a pequena placa lateral. Membrana inte:-segmentar castanho-escura, aveludada, intercalada com mancha clara que vai do segmento II ao IV. Pós-abdômen castanho-claro, formado pelo tergitos VIII e IX, com pêlos claros e curtos e pêios mais longos que se dispõe nas margens. Esses segmentos são encurvados no sentido ventral, a borda posterior do segmento IX é ocupada pelos cercos bem constituías. Genitália (Fig. 10): Esternito IX em forma de semicirculo dorsal, cujas extremidades alongam-se no sentido basal, que se unem $\in m$ posição mediana, formando uma placa encurvada consistente de estrias transversas (Fig. 10a). A porção inferior dessas traves alonga-se moderadamente articulando-se com as extremidades basais do tergito IX, próximo à porção basal do anel, estendendo-se distalmente em duas traves laterais, que sustentam a camada transparente, ornamentada, com finos pêlos laterais. Apódema do duto (Fig. 10b) semi-esférico na base, onde se observa uma glândula em cacho, pedúnculo bem constituído e escierosado, a ex. tremidade oposta alarga-se em forma de leque, tornando-se mais transparente e menos esclerosada. Apódema do pênis alargado na porçāo proximal com estrias medianas transversais: a porção distal alonga-se em forma de calha, que se distende próximo ao ápice, lateralmente, em duas traves que sustentam na extremidade as pinças fálicas, pequenas placas encurvadas, divergentes e com espinhos superficiais. A porção distal da teca une-se à porção basal do pênis, por uma jlaca em forma de anei semicircular de posição dorsal. A porção proximal do falo, de forma tubular, é sustentada por uma farpa longitudinal esclerosada, onde se prende a me:nbrana ornamentada em toda extensão; ao 
longo da membrana corre internamente o ducto ejaculador de diâmetro moderado, consistente, com finas estrias transversas, que atravessa uma ampola esclerosada de forma bem característica onde se continua para a porção terminal do falo, muito alongada. Neste segmento (Fig. 12), a membrana é sustentada por uma estria esclerosada robusta na base, que vai se afilando e não chega a atingir a extremidade, onde a membrana é finamente ornamentada, formando próximo ao ápice ligeira dilatação. Quando em repouso esse segmento fica disposto em espiral.

FÊMEA - Comprimento em média de $10,83 \mathrm{~mm}$. Morfologia idêntica a do macho. Apresenta, porém, corpo ligeiramente mais longo, conseqüentemente asas mais longas e largas, em méćia $7,6 \mathrm{~mm}$ de comprimento por $2,70 \mathrm{~mm}$ de largura. Abdômen (Fig. 13) constituído por seis segmentos. Tergitos: castanhoescuros semelhantes aos do macho. Esternitos: do I ao $\mathrm{V}$ alongados, sendo o esternito $\mathrm{VI}$ o mais desenvolvido e alargado. Os restantes dos segmentos transformados em ovipositor de cor castanho-enegrecida brilhante, com micropilosidade e pruinosidade prateada na face dorsal, que é nitidamente encurvada. Esclerito basal e ventral do ovipositor de forma bem característica, preso à membrana, onde se evidencia um par de espiráculos; a membrana se continua lateralmente até a altura média do segmentos (Fig. 14). Genitália (Fig. 15); espermatecas presas a um ducto volumoso, fortemente enrugado; na extremidade basal, o ducto forma uma ampola que reduz o diâmetro e o divide em dois ramos, cada um sustentando na extremidade uma espermateca desenvolvi. da, alongada, de parede interna consistente e estriada, com superfície espinhosa e ligeira invaginaçào no ápice. Próximo à extremidade distal do ducto, parte um tubo de diâmetro menor e parede estriada, que sustenta uma espermateca rudimentar, alongada, de parede consis tente. espinhosa e ricamente ornamentada, (Fig. 16). A porção distal do ducto torna-se dilatada, membranosa, ornamentada, tendo na face ventral duas placas longitudinais esclerosadas que sustentam os cercos, cuja forma é bem definida, com espinhos característicos em número, disposição e tamanho para a espécie

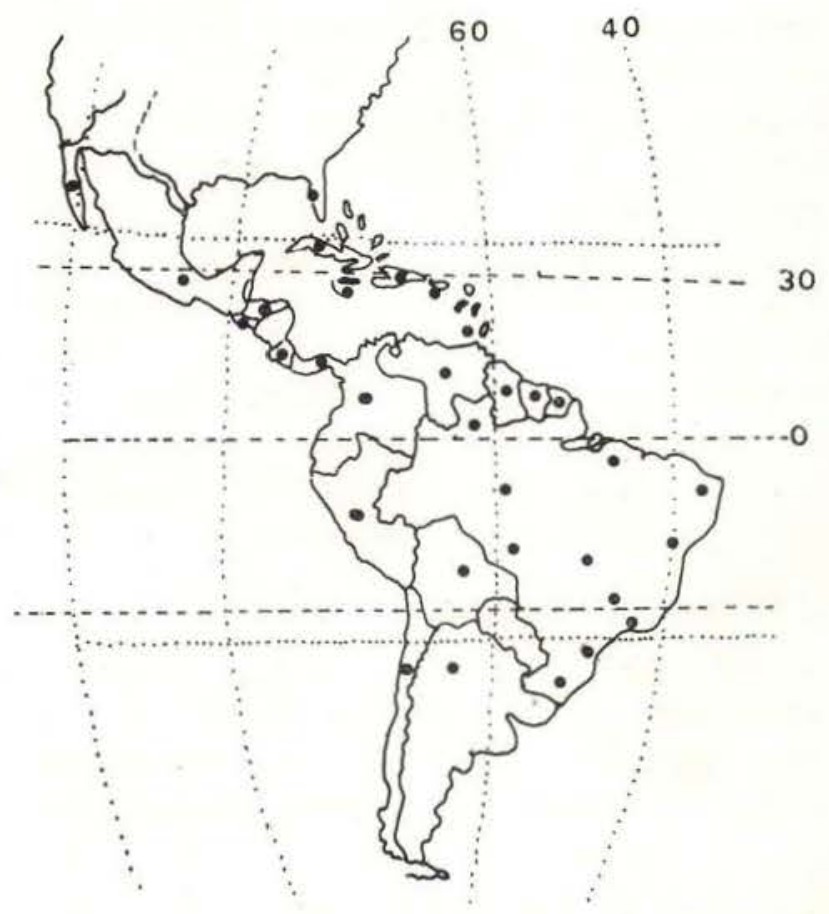

Fig. 17 - Distribuição geográfica conhecida de Taeniaptera lasciva.

DistribUição GeOgRÁfica - México; Hon duras; Costa Rica; Panamá; Colômbia; Venezuela; Suriname; Guiana; Guiana Francesa; Antilhas: Trinidad, São Domingos, Jamaica, Cuba, São Salvador; Brasil (Roraima ao Rio Grande do Sul): Peru; Bolivia; Paraguai; Norte da Argentina: Formosa e Tucumã; Chile; EUA: Flórida, Califórnia.

MATERIAL EXAMINAdo - Amazonas - Manaus - 1 macho $R$. Ducke, A. Faustino, 7.3.1968; 1 macho Pomar-Lages, E. V. Silva, 24.4.1968; 1 macho Estrada AM. 1. Km 104, L. P. Albuquerque, A. Faustino, 8.5.1968; 1 macho R. Ducke, L. P. Albuquerque, 13.5.1968; 1 fêmea Colônia Santo Antônio, A. Faustino, 15.6.1968; 1 macho Estrada do Aleixo-Lajes, E. V. Silva, 28.5.1968; 1 macho e 1 fêmea R. Ducke, E. V. Silva, 5.1968; 1 fêmea Estrada AM.1. Km 182, A. Faustino, E. V. Silva, 3.7.1970; 2 machos Colônia Santo Antônio, T. Pimentel, 8.7.1970; 1 fêmea Estrada AM.1. Km 31, E. Castellon, 30.4.76; 1 fêmea Campus do INPA, L. P. Albuquerque, 7.76; 2 machos BR-174, Km 220, N. D. Penny, 13.5.77; 1 fêmea Tabatinga, F. B. Almeica e A. Faustino, 3.9.69; 
Pará - 1 fêmea Posto Santana, J. I. B. Bechi. nê, 4.7.61; 1 fêmea Belém-Brasília $\mathrm{Km} 90, \mathrm{~T}$. Pimentel, 24.7.72; 1 macho, Belém-Brasília $M^{a}$ Helena, 25.7.72; 1 macho Belém-Brasília, Km 90, T. Pimentel, 21.4.73; 1 macino, Salinas Agri-Sol, I. Shak, 26.7.73; 1 macho Belém-Brasília, Km 90, Ma Helena 25.7.74; 1 têmea, Belém-Brasília, $\mathrm{Km}$ 90, F. Torres, 26.2.76; Distri to Federal - 1 macho e 3 fêmeas, Brasília, $H$. S. Lopes, 1.61; Mato Grosso - 1 fêmea Ari. puanã, L. P. Albuquerque, 23.1.76; Minas Gerais - 1 macho Cameriqueira Lopes \& Gomes, 2.1.41; 1 macho Governador Valadares, Oliveira, 5.9.43; Rio de Janeiro - Vassouras, D. Machado, 1940; 1 macho e 1 fêmea Cascaaura. Oliveira, 9.1.40; São Paulo -2 machos 1 fêmea, Barueri, K. Lenko, 7.4.57; Rio Grande do Sul - 1 macho Porto Alegre, P. Cabral, 1954.

\section{DISCUSSÃo}

Os caracteres usados nas descrições originais e redescrições das espécies já conhecidas de Taeniaptera prendem-se quase totalmente à morfologia externa, tais como: coloração, manchas da asa, comprimento relativo das nervuras, anéis dos fêmures e quetotaxia.

Hennig (1934) foi o primeiro a apresentar:

- um modelo esquemático da genitália dos machos (Fig. 1-3) com a nomenclatura das peças usadas em parte neste trabalho;

- forma geral do abdômen do macho (Fig. 4-6) de Taeniapterinae e Trepidariinae, onde se observa modificações no comprimento e forma dos tergitos e esternitos, especialmente no $\mathrm{V}$ esternito do macho (processo copulatório) e hipopígio;

- o estudo comparativo da morfologia do aedeago em espécies de nove gêneros (Fig. 7-18);

- modelo dos fórcipes inferiores em espécies de três subfamílias de Micropezidae. Nos Taeniaptera porém essas peças inexistem;

- forma geral do abdômen de três subfamílias evidenciando-se ligeiras modificaçōes na morfologia externa que permitem separá-las.

Hennig (1936) apresenta desenhos comparativos da cabeça, asa, escutelo e $\mathrm{V}$ esternito de espécies de Taeniaptera.
Para Aczel (1949), Taeniaptera na América do Sul é o gênero de Micropezidae que reune o maior número de caracteres arcáicos. Em oposição a esse argumento Hennig (1934) considera o desaparecimento dos fórcipes superiores e inferiores, neste grupo, um caráter evoluído.

$T$. lasciva apresenta um conjunto significativo de caracteres evoiuidos. Cresson (1930) cita $T$. trivitatta na sinonímia de $T$. lasciva Johnson (1890) (nec. Fabr.). Embora não se possa discutir para uma decisão definitiva a individualidade dessas espécies, por não se ter examinado os "tipos". Considera-se, porém, fora de cogitação, uma sinonímia entre $T$. lasciva (Fabr.) e $T$. trivitatta (Macq.), com base em dois fatores de relevante importância: a) Morfologia - apófise do $\mathrm{V}$ esternito é ausente em lasciva, enquanto trivitatta apresenta o caráter (Hennig, 1936: Taf. 3, fig. 21 e 26 e Cresson, 1938: PI. XIII, fig. 31 e 32); b) Distribuição geográfica que reforça o primeiro argumento, $T$. lasciva é neártica e neotropical, estendendo-se do sui da Califórnia ao norte da Argentina, T. trivitatta é a única espécie do gênero assinalada na bibliografia com distribuição apenas neártica.

$\Delta$ través do estudo da anatomia da genitália a espécie $T$. lasciva apresenta a porção terminal do aedeago em espiral e medindo duas vezes o tamanho da porção basal.

\section{Conclusã̃o}

Pelos resulatdos das consultas à bibliografia do grupo e de exame de exemplares, atribui-se como caracteres válidos para o estudo da sistemática: distribuiçăo dos anéis dos fêmures, tonalidade de coloração e quetotaxia, como meios auxiliares na separação das espécies pela morfologia externa; considera-se de relevante importância o estudo da genitália de dipteros, em ambos os sexos, para decisões ac nivel de espécie; a forma, tamanho e tonalidade das manchas das asas que são variáveis entre as espécies e com gradação de manifestações dentro da mesma espécie, não chegam a invalidar o caráter na identificação aos Tae niaptera. 
T. lasciva Fabr. (1798) é uma espécie bem caracterizada pela morfologia externa. Esses caracteres são agora reforçados pelo conhecimento da anatomia da genitália dos dois sexos e apresentados nos desenhos da morfologia da espécie.

\section{AgRADECIMENTOS}

Aos Drs. Hugo de Souza Lopes, Paulo F. Bührnheim, Norman D. Penny e Victor Py-Daniel, pela contribuição com sugestões, na correção do texto, na bibliografia e na versão do Resumo.

\section{SUMMARY}

This work is an evaluation of micropezid systematics in view of the evolutionary position of the family, especially as applied to the subfamily Taeniapterinae. The study of the genus Taeniaptera begins with thosa species which occur in central Amazonia, based on differential characters of male genitalia and females. The identification of specimens was made using the key of Hennig 1934:47-77 and comparing characters of these specimens vith the original description of each species and with material identified by dipterologist $\mathrm{Dr}$. H. S. Lopes. The morphology, genital anatomy, and distribution of Taeniaptera lasciva (Fabr., 1798) is also included. This species presents a closed and stalked posterior cell of the wing, and simple male fifth sternite.

\section{BIBLIOGRAFIA}

ACZEL, M.

1949a-- Notes on "Tylidae" II. Acta Zool. Lilloana 8: 219-280. pls. 1-2.

1949b- Catálogo de la familia de las "Tylidae" (Calobatidae + Micropezidae + Neriidae, Diptera) en la region neotropical. Acta Zool. Lilloana 8: 309-389.

1951 - Division sistemática de las "Tanyperidiformes". Acta Zool. Lilloana, 11: 483-589.

Cresson, E.T., JR.

1930 - Notes on and descriptions of some neotropical Neriidae and Micropezidae (Diptera) Trans. Amer. Ent. Soc. 56: 307-363.

1938 - The Neriidae and Micropezidae of America North of Mexico (Diptera) Trans. Amer. Ent. Soc., 64: 293-366, pls. III.

CZERNY, L.

1930 - Die Musca annulata F. (Diptera-Micropezi dae) Mitteil. Deutsche. Ent. Ges. 1 (8): $117-120$.
ENDERLEIN, G.

1922 - Klassifikation der Micropeziden. Arch. Na turg. $88(\mathrm{~A}):$ 140-229.

FABRICIUS, J.C,

1798 - Supplementum entomologie systematicae 572pp. Hafniae (=Copenhagen).

1805 - Systema antliatorum secundum ordines, ge nera, species $373+30 \mathrm{pp}$. Brunsvigae $\mathrm{l}=$ Brunswick)

FisCHER, C.R.

1932 - Contribuição para conhecimento da metamorphose e posição systematica da familia Tylidae (Micropezidae, Dipt.,) Rev. de Ent 2: $15-24$.

FREY, R.

1927 - Zur Systematik der Diptera Haplostomata III. Fam. Micropezidae. Notulae Ent. 7: 65-76.

GuérIN - MENÉviLle, F.E.

1844 - Douzième Ordre. Les Diptères. pp. 531. 559 (livro 50), in Iconographie du règne animal de G. Couvier 3 (Texte explicatif. Insects) 576pp. $(=$ livr. 45-50). Paris.

HENDEL, $\mathrm{F}$.

1922 - Einige neue Micropeziden. Deutsch. Ent. Zts. 231-235.

1933a- Ueber oinige Typen Wiedemann's und Schiner's von acalyptraten Musciden aus Südamerika, nebst einige verwandten Arten (Dipt.). Rev. de Ent. 3: 58-83.

Hennig, W.

1934 - Zur Kenntnis der Kopulationsorgane der Tyliden (Micropeziden) Dip. Acalypt. Zool. Anzg. Helt $3 / 4$. Mit 25 (Abbildoungen) pgs. 67-76.

1934 - Revision der Tyliden (Dipt. Acalypt.) I. Teil: Die Taeniapterinae Amerikas. Stettin. Ent. Ztg. 95: 55-198, 294-330

1935 - Do. Idem 96: 27-67.

1936 - Do. II Teil: Die ausseramerikanischen Taeniapterinae, die Trepidariinaz un Tylinae. Allgemeines über die Tyliden Konowia 14: 637-231.

1937 - Nachträge zur "Revision der Tyliden" Stett. Ent. Zeitg. 98: 46-50

1938 - Neue Beitrage zur Systematik der Richardiiden und Tyliden (Diptera-Acalyptrate) Arb Morph. Taxonom. Ent. Berlin - Dahlen 5 : 8-15.

1973 - Handbook of Zoology, 31 Diptera p. 32.

INTERNATIONAL COMMISSION FOR ZOOLOGICAL NOMENCLATURE

1956 - Officia! list of family - Group Names in Zoology, Direction 41, apud Zoological Re. cord, 93 (13): 465 . 
JoHNSON, C.W.

1894 - List of Diptera of Jamaica with descriptions of new species. Proc. Acad. Nat. Sci Philad., 271-281.

LOEW, $\mathrm{H}$.

1866 - Diptera Americae septentrionalis indigena Centuria septima Berlin. Ent. Zts. 10: 1-54.

MACQUART, J.

1835 - Histoire naturelle des insectes Diptères 2: 703pp., pls. 13-24 (In: Reret, N.E. ed. Collection des Suites à Buffon.

ROHDENDORF, B.B.

1974 - The historical Development of Diptera, p. 110.
RONDANI, C.

1850 - Osservazioni sopra alquante specie di Esapodi Ditteri del Museu Torinese. Nuovi Ann. Sci. Nat. (Bologna) (3) 2: 165-197 pl. 4 .

STEYSKaL, G.C.

1968 - A catalogue of Diptera of the Americas South of the United States. Family Micropezidae, Dep. Zool Sec. Agric. Est. São Paulo. 48: 1-33.

WULP, F.M. VAN DER

1897 - Group Calobatinae, in F.D. Godman \& O. Salvin, eds. Bilogia Centrali-Americana, Zool. Ins., Diptera 2: 363-376, pl. 9.

(Aceito para publicação em $28 / 07 / 80$ ) 